\title{
Successful treatment with nilotinib for molecular relapse of chronic myeloid leukemia after allogeneic hematopoietic stem cell transplantation in the accelerated phase
}

Shuichi Ota, Kiyotoshi Imai, Takahide Ara, Reiki Ogasawara, Koichiro Minauchi, Teiichi Hirano, Masahiro Ogasawara, Yoshio Kiyama, Naoki Kobayashi, and Masahiro Imamura

Department of Hematology, Sapporo Hokuyu Hospital, Sapporo

Although allogeneic hematopoietic stem cell transplantation (allo-HSCT) is recommended for patients with chronic myeloid leukemia (CML) in the advanced phase, but the prognosis of patients who relapsed after allo-HSCT has been poor. An 18-year-old male CML patient achieved complete molecular response (CMR) with nilotinib following molecular relapse soon after allo-HSCT from an HLA-matched sibling donor in the accelerated phase. There was no acute graft-versus-host disease and minimal residual disease levels gradually increased after allo-HSCT. Nilotinib was prescribed at $800 \mathrm{mg} /$ day and started again when molecular relapse was observed at 6 months after HSCT. He maintains complete donor-type chimerism of myeloid cells and CMR after 18 months of nilotinib treatment and 2 years after allo-HSCT without any donor lymphocyte infusion. Although he experienced a sudden onset of intramuscular hemorrhage, probably associated with nilotinib administration combined with an anti-fungal agent, before allo-HSCT, no adverse events were observed in association with nilotinib treatment for molecular relapse after allo-HSCT. In conclusion, nilotinib is an effective and safe agent for molecular relapse after allo-HSCT for CML patients in the advanced phase. (Journal of Hematopoietic Cell Transplantation 2(2): 56-60, 2013.)

\section{Introduction}

Recently, the standard therapy for patients with newly diagnosed chronic myeloid leukemia (CML) in the chronic phase (CP) has consisted of treatment with one of three tyrosine kinase inhibitors (TKIs) such as imatinib, dasatinib, or nilotinib. However, patients with CML in the advanced phase (accelerated phase $[\mathrm{AP}]$ and blast phase [BP]) respond particularly poorly to imatinib, with imatinib-resistance developing in $70 \%$ of AP patients and $90 \%$ of BP at 4 years. ${ }^{1}$ Therefore, allogeneic hematopoietic stem cell transplantation (allo-HSCT) still has an important role in the management of advanced phase CML. ${ }^{2,3}$ Relapse after allo-HSCT for CML patients has been treated with donor lymphocyte infusion (DLI), imatinib, or additional transplantation, but each treatment has certain drawbacks. Nilotinib (Tasigna; Novartis pharmaceuticals corporation, USA) is a second-generation
BCR-ABL1 TKI, with increased potency and improved pharmacological properties compared with imatinib. Phase II studies of nilotinib in patients with CML in the advanced phase with imatinib failure demonstrated that nilotinib was associated with 24-month overall survival rates of $70 \%$ and $27 \%$ in AP and BP, respectively. ${ }^{4,5}$ Furthermore, nilotinib showed efficacy in patients with $\mathrm{CML}-\mathrm{BP},{ }^{5}$ but has shown limited long-term survival of these patients without HSCT. We experienced a patient who had a CML molecular relapse early after allo-HSCT. He achieved a complete molecular response $(\mathrm{CMR})$ after treatment with nilotinib alone and is still in CMR at 2 years after allo-HSCT without DLI.

\section{Case report}

An 18-year-old male developed persistent fever, and a blood examination revealed hyperleukocytosis with a white

Submitted January 25, 2013; accepted March 26, 2013.

Key words: Nilotinib, chronic myeloid leukemia, hematopoietic stem cell transplantation

Correspondence: Shuichi Ota, M. D. Department of Hematology, Sapporo Hokuyu Hospital Higashi-Sapporo 6-6, Shiroishi-ku, Sapporo 003-0006, Japan. E-mail:shuota@hokuyu-aoth.org. dx.doi.org/10.7889/hct.2.56 C The Japan Society for Hematopoietic Cell Transplantation. 
blood cell count of $13.1 \times 10^{9} / \mathrm{L}$ with $8 \%$ blast cells and $25 \%$ basophils. Bone marrow examination showed enhanced total nucleated cells, $520 \times 10^{9} / \mathrm{L}$, including $3.6 \%$ blast cells, and the Philadelphia $(\mathrm{Ph})$ chromosome was identified in all metaphase spreads, while an additional clonal chromosome abnormality, $\mathrm{t}(7 ; 8)$ (q22;q13), was also identified. The patient was diagnosed with $\mathrm{CML}-\mathrm{AP}$ with warning factors using The European LeukemiaNet (ELN) criteria. ${ }^{2} \mathrm{He}$ received imatinib therapy at $600 \mathrm{mg} /$ day and achieved a complete hematological response after 18 days of imatinib treatment. After 2 months of treatment with the initial dose of imatinib, he received imatinib at a reduced dose of 400 $\mathrm{mg} /$ day due to severe myalgia. Imatinib was continuously administered. Although the $\mathrm{Ph}$ chromosome was decreased to $6 / 24$ cells in metaphase spreads and $27.3 \%$ by fluorescence in situ hybridization in the optimal response after 3 months of imatinib $400 \mathrm{mg} /$ day monotherapy, imatinib was replaced with nilotinib at $800 \mathrm{mg} /$ day due to its unsatisfactory effect. Thereafter, he achieved a cytogenetic response after 1 month of nilotinib treatment. Due to the risk factors presented (clonal chromosome abnormalities), the patient was scheduled for his allo-HSCT from an HLA-matched sibling donor in November 2010. However, sudden-onset intramuscular hemorrhage was observed 10 days before the first scheduled allo-HSCT, which was possibly associated with the nilotinib administration combined with the use of an anti-fungal agent. Prophylaxis against fungal infection was performed with an itraconazole oral solution at $200 \mathrm{mg} /$ day 2 weeks before allo-HSCT. Since blood examination revealed a platelet count of $18.1 \times 10^{9} / \mathrm{L}$ at hemorrhage onset, the platelet count did not seem to be related to intramuscular hemorrhage. He received no anticoagulation therapy. Thereafter, because his hemorrhage improved after nilotinib was stopped, we performed the first scheduled allo-HSCT one month later using micafungin at $50 \mathrm{mg}$ /day 5 days before allo-HSCT.

The conditioning regimen consisted of total body irradiation with a total dose of 12 Gy divided into 6 fractions and cyclophosphamide at a total dose of $120 \mathrm{mg} / \mathrm{kg}$ divided over 2 days (Figure 1). Nilotinib was stopped one day before the start of the conditioning regimen. Bone marrow cells collected from the HLA-matched sibling donor were infused without ex vivo manipulation. The number of infused nucleated cells was $3.17 \times 10^{8}$ cells $/ \mathrm{kg}$ of recipient body weight. Prophylaxis against graft-versus-host disease (GVHD) was performed with the continuous infusion of cyclosporin at $3 \mathrm{mg} / \mathrm{kg} /$ day and short-term methotrexate at 10 $\mathrm{mg} / \mathrm{m}^{2}$ on day 1 and $7 \mathrm{mg} / \mathrm{m}^{2}$ on days 3 and 6 . Neutrophil engraftment, defined as the first of 3 consecutive days with an absolute neutrophil count of at least $0.5 \times 10^{9} / \mathrm{L}$, was documented on day 19. Acute GVHD was not observed until 6 months after HSCT. Qualitative PCR for the BCR-ABL fusion transcript was persistently negative at $<1 \times 10^{2}$ copies/ $\mu \mathrm{g}$ RNA after HSCT. However, the MRD assessed by a $\mathrm{BCR}-\mathrm{ABL}$ transcription-mediated amplification and hybridization protection assay (TMA-HPA) was positive at 27 copies/ $0.5 \mu \mathrm{g}$ RNA on day 40 after HSCT. ${ }^{6}$ Cyclosporin was rapidly reduced to 20 to $50 \mathrm{mg}$ every 2 weeks and stopped to harness the graft-versus-leukemia (GVL) effect on day 140 after HSCT; even so, MRD levels increased in the peripheral blood. Nilotinib at $800 \mathrm{mg}$ /day was started again as soon as molecular relapse was confirmed by the increased level of MRD up to 393 copies/ $0.5 \mu \mathrm{g}$ RNA as determined by TMA-HPA on day 154 after HSCT. Thereafter, these MRD levels were quickly reduced by nilotinib treatment. Because chronic GVHD of the limited type was observed in the skin from 2 months after cyclosporin was stopped, low dose tacrolimus at $0.2 \mathrm{mg} /$ day was started on day 238 after HSCT. Nilotinib was well tolerated and CMR defined as $<5$ copies/0.5 $\mu \mathrm{g}$ RNA by TMA-HPA was confirmed on day 258 after HSCT, 2 months after the start of nilotinib treatment. Chronic GVHD improved quickly with low dose tacrolimus, and remained controllable. He maintains complete donortype chimerism of myeloid cells and CMR with 18 months of nilotinib administration following allo-HSCT without DLI.

\section{Discussion}

The ELN has recommended a treatment algorithm for CML as follows; allo-HSCT for instances of drug therapy failure, and for patients in the advanced phase. ${ }^{2}$ For a minority of patients who fail TKI or progress to advanced phase disease, allo-HSCT still has an important role in the management of advanced phase CML. ${ }^{7}$

In the present case, molecular relapse was detected by TMA-HPA shortly after allo-HSCT, but CMR was achieved with nilotinib alone without DLI. Sustained CMR has been observed for more than 18 months following allo-HSCT. The clinical course strongly suggested that nilotinib had a much stronger anti-leukemic activity than conditioning chemotherapy for allo-HSCT in this patient. He was able to tolerate nilotinib at $800 \mathrm{mg} /$ day after allo-HSCT and this may 


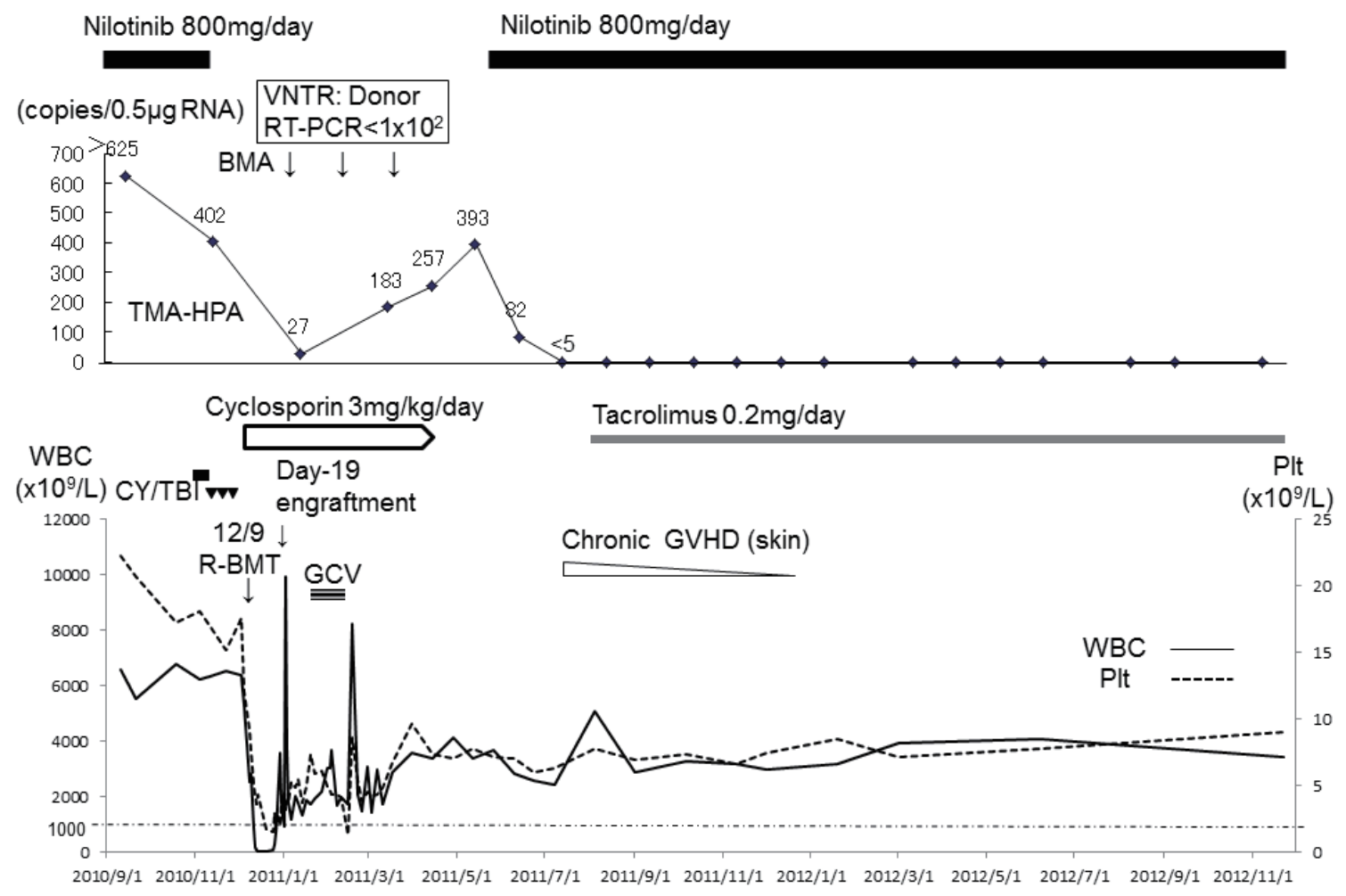

Figure1. Clinical course of a patient with CML in the accelerated phase who had a molecular relapse after allo-HSCT.

In June 2010, he started to receive imatinib and achieved a complete hematological response after 18 days of imatinib treatment. In September 2010, we began nilotinib treatment due to intolerance of imatinib. Thereafter, he achieved a cytogenetic response after one month of nilotinib treatment. The patient had a bone marrow transplant (BMT) performed from an HLA-matched sibling donor in December 2010. Qualitative PCR for the BCR-ABL fusion transcript was persistently negative at $<1 \times 10^{2}$ copies/ $\mu \mathrm{g}$ RNA after allo-HSCT. However, transcription-mediated amplification and hybridization protection assay (TMA-HPA) levels gradually increased after HSCT. Nilotinib prescribed at $800 \mathrm{mg} /$ day was started again as soon as molecular relapse was observed at 6 months after HSCT. After nilotinib administration, the corrected ratio of BCR-ABL with TMA-HPA levels was dramatically reduced and a second molecular response after allo-HSCT was achieved and this condition was sustained for more than 18 months. BMA, bone marrow aspiration; $\mathrm{CY}$, cyclophosphamide; TBI, total body irradiation; R-BMT, related-bone marrow transplantation; GVHD, graft-versus-host disease; VNTR, variable number of tandem repeats; GCV, ganciclovir.

have partly contributed to his ability to maintain a molecular response. Although there are a few reports of nilotinib treatment for recurrent CML after allo-HSCT, 24-month follow-up of a large, phase II study of CML-AP patients treated with nilotinib $400 \mathrm{mg}$ twice daily reported favorable long-term outcomes and a manageable safety profile of nilotinib in an FDA report. ${ }^{4,8}$ Therefore, we decided on the same dose of nilotinib in a phase II study of CML-AP.

DLI has been used in the treatment of patients with CML relapse after allo-HSCT, and showed effectiveness, but can also cause GVHD occurrence. DLI is associated with $20 \%$ to $40 \%$ rates of grades III-IV GVHD, $30 \%$ to $40 \%$ rates of significant myelosuppression, and a $20 \%$ rate of treatmentrelated deaths. ${ }^{9}$ Generally, immunosuppressive treatment was reduced and stopped to induce the GVL effect for relapse of leukemia after allo-HSCT. MRD levels continued increasing even after cyclosporin was stopped in this patient. Chronic GVHD of a limited type occurred in the skin 2 months after cyclosporin administration was stopped. Although we did not perform DLI for this patient with chronic GVHD, combination therapy with TKI and DLI may be a candidate treatment for patients who fail to achieve CMR with TKI therapy alone.

Although imatinib effectively controlled CML relapse 
after allo-HSCT, it was associated with adverse effects including myelosuppression and recurrence of severe GVHD. ${ }^{9}$ Hess et al. reported that imatinib treatment was well-tolerated and highly efficacious in MRD after alloHSCT. $^{10}$ It is important that MRD be found in early relapse by regular monitoring of the molecular response after alloHSCT in CML. Wright et al. also retrospectively reviewed patients treated with imatinib and/or dasatinib for relapsed CML after HSCT, and concluded that TKI therapy can induce durable molecular responses for relapsed CML in the advanced phase. ${ }^{11}$

In addition, this patient experienced intramuscular hemorrhage before HSCT. Although the effects of the prophylaxis agents against fungal infection may play a major role in the metabolism of nilotinib, the sudden-onset intramuscular hemorrhage was atypical. Previous in vitro studies indicated that nilotinib metabolism is primarily mediated by cytochrome P450 3A4 (CYP3A4). In a study to investigate the effect of CYP3A4 inhibition on nilotinib pharmacokinetics, ketoconazole increased the Cmax and AUC of nilotinib, compared with nilotinib alone. ${ }^{12}$ Also, Quintás-Cardama et al. reported that TKIs induced platelet dysfunction in patients with CML. ${ }^{13}$ Although the blood concentration level of nilotinib could not be examined, we considered that the use of nilotinib with azole agents may have adversely affected the coagulation in this patient. During treatment with nilotinib, it was not clear how often bleeding occurred, and why this episode of bleeding was observed in muscle. However, these results indicate that concurrent use of CYP3A4 inhibitors, for example itraconazole, fluconazole, and voriconazole, necessitate dosage adjustments of nilotinib in HSCT and should be avoided when possible.

Imatinib is effective at reducing the tumor burden, but it does little to eradicate leukemic stem cells, ${ }^{14}$ so the disease typically recurs after stopping the drug, necessitating other strategies such as DLIs. It is unclear whether nilotinib can induce cure of CML-CP and also eradicate leukemic stem cells. Recently, a goal of CML therapy has been to focus on treatment cessation studies of patients in long term CMR by second-generation TKIs. However, we should not attempt to discontinue TKIs in advanced CML patients with a second sustained CMR after allo-HSCT outside of clinical trials.

In conclusion, nilotinib induced molecular remission in a patient who had an MRD relapse of CML shortly after alloHSCT. This suggests that nilotinib has a strong anti-leukemic activity against advanced CML, and therefore, the use of nilotinib not only as a salvage therapy, but also as prophylaxis against relapse, may be beneficial for patients with advanced CML after allo-HSCT. A standardization of the posttransplant application of TKIs regarding intervals from SCT, dosages, treatment duration, and the combination with DLI has not yet been confirmed. Thus, multicenter clinical trials should be performed focusing on such high risk CML patients in the near future.

\section{Conflict of interest}

The authors declare no conflict of interest.

\section{Author contributions}

SO wrote the manuscript. SO, KI, TA, RO, KM, TH, MO, YK and NK were responsible for patient care. MI reviewed the manuscript. All authors read and approved the final manuscript.

\section{References}

1. Jabbour E, Cortes J, Santos FP, et al. Results of allogeneic hematopoietic stem cell transplantation for chronic myelogenous leukemia patients who failed tyrosine kinase inhibitors after developing BCR-ABL1 kinase domain mutations. Blood. 2011; 117: 3641-3647.

2. Baccarani M, Cortes J, Pane F, et al. Chronic myeloid leukemia: an update of concepts and management recommendations of European LeukemiaNet. J Clin Oncol. 2009; 27: 6041-6051.

3. Radich J. Stem cell transplant for chronic myeloid leukemia in the imatinib era. Semin Hematol. 2010; 47: 354-361.

4. le Coutre PD, Giles FJ, Hochhaus A, et al. Nilotinib in patients with $\mathrm{Ph}+$ chronic myeloid leukemia in accelerated phase following imatinib resistance or intolerance: 24-month follow-up results. Leukemia. 2012; 26: 1189-1194.

5. Giles FJ, Kantarjian HM, le Coutre PD, et al. Nilotinib is effective in imatinib-resistant or -intolerant patients with chronic myeloid leukemia in blastic phase. Leukemia. 2012; 26: $959-962$

6. Sumi M, Tauchi T, Sashida G, et al. Clinical usefulness of transcription-mediated amplification and hybridization protection assay in imatinib-treated chronic myelogenous leukemia patients. Clin Lab Haematol. 2005; 27: 416-417. 
60 Ota et al.

7. Venepalli N, Rezvani K, Mielke S, Savani BN. Role of alloSCT for CML in 2010. Bone Marrow Transplant. 2010; 45 : $1579-1586$

8. Hazarika M, Jiang X, Liu Q, et al. Tasigna for chronic and accelerated phase Philadelphia chromosome-positive chronic myelogenous leukemia resistant to or intolerant of imatinib. Clin Cancer Res. 2008; 14: 5325-5331.

9. Kantarjian HM, O' Brien S, Cortes JE, et al. Imatinib mesylate therapy for relapse after allogeneic stem cell transplantation for chronic myelogenous leukemia. Blood. 2002; 100: 1590-1595.

10. Hess G, Bunjes D, Siegert W, et al. Sustained complete molecular remissions after treatment with imatinib-mesylate in patients with failure after allogeneic stem cell transplantation for chronic myelogenous leukemia: results of a prospective phase II open-label multicenter study. J Clin Oncol. 2005; 23: 7583-7593.
11. Wright MP, Shepherd JD, Barnett MJ, et al. Response to tyrosine kinase inhibitor therapy in patients with chronic myelogenous leukemia relapsing in chronic and advanced phase following allogeneic hematopoietic stem cell transplantation. Biol Blood Marrow Transplant. 2010; 16: 639-646.

12. Tanaka C, Yin OQ, Smith T, et al. Effects of rifampin and ketoconazole on the pharmacokinetics of nilotinib in healthy participants. J Clin Pharmacol. 2011; 51: 75-83.

13. Quintás-Cardama A, Han X, Kantarjian H, Cortes J. Tyrosine kinase inhibitor-induced platelet dysfunction in patients with chronic myeloid leukemia. Blood. 2009; 114: 261-263.

14. Graham SM, Jørgensen HG, Allan E, et al. Primitive, quiescent, Philadelphia-positive stem cells from patients with chronic myeloid leukemia are insensitive to STI571 in vitro. Blood. 2002; 99: 319-325. 